\title{
Celecoxib and acetylbritannilactone interact synergistically to suppress breast cancer cell growth via COX-2-dependent and -independent mechanisms
}

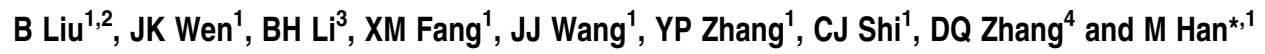

The use of celecoxib is associated with a significant decrease in breast cancer risk. However, the long-term use of high-dose celecoxib might be limited owing to cardiovascular side effects. In this study, we found that acetylbritannilactone (ABL), extract from a Chinese medicinal herb, could reduce celecoxib dose and potentiate the growth-inhibitory effect in breast cancer cells. $A B L$ enhanced the apoptotic effect of celecoxib in COX-2-expressing cells, but had little effect in COX-2-negative cells. The apoptosis induced by the combination treatment disappeared when COX-2 was knocked down, whereas the lack of apoptotic effects in COX-2-negative cells was reversed after COX-2 transfection. However, the combination treatment induced a $G_{0} / G_{1}$ phase arrest independent of whether or not the cells expressed COX-2. The $G_{0} / G_{1}$ arrest was attributed to a decreased expression of cyclinD1, cyclinE, CDK2 and CDK6, especially the upregulation of p21. In addition, inhibition of Akt and p38 signaling pathways was required by the synergism, as the constitutively active Akt and p38 protected cells against apoptosis and cell cycle arrest induced by the combination treatment. In vivo, administration of celecoxib and ABL were more effective than the individual agents against xenograft tumor growth. Thus, our data suggested that the combinatorial approach of celecoxib and ABL might be helpful for breast cancer treatment.

Cell Death and Disease (2011) 2, e185; doi:10.1038/cddis.2011.64; published online 28 July 2011

Subject Category: Cancer

Breast cancer is the most common cancer among women in both developed and underdeveloped countries. Abnormally elevated expression of cyclooxygenase-2 (COX-2) has been frequently observed in breast cancer tissues and some researches support the concept that COX-2 could provide an early target for breast cancer prevention. ${ }^{1-3}$ Previous studies demonstrated that administration of non-steroidal anti-inflammatory drugs (NSAIDs), such as aspirin, ibuprofen, naproxen, piroxicam or indomethacin, had significant chemopreventive effects against the development of breast cancer. $^{4,5}$ On the contrary, the long-term use of them may be limited owing to serious adverse effects among users, such as ulcer complications, bleeding, cardiovascular toxicity, platelet dysfunctions and renal toxicity, ${ }^{6,7}$ as a result of interference of COX-1-derived homeostatic functions. Therefore, it is rational to search for specific inhibitors of COX-2 as potential candidates for use as chemopreventive agents. Celecoxib, the first selective COX-2 inhibitor approved by the U.S. Food and Drug Administration, is a widely marketed antiinflammatory drug with improved safety unlike traditional NSAIDs ${ }^{8}$ and exerts potent anticancer effects in various tumor types, including the colon, skin, prostate, lung and breast. ${ }^{9-14}$ However, the exact molecular mechanisms involved are not yet defined. Many lines of evidence from in vitro studies and animal models indicated that celecoxib exerted its effect by preventing COX-2 protein expression and prostaglandin $E_{2}$ $\left(\mathrm{PGE}_{2}\right.$ ) synthesis. ${ }^{9,10}$ Moreover, other studies suggested that celecoxib might suppress cell proliferation and carcinogenesis by downregulating multidrug resistance-associated protein expression ${ }^{12}$ or inhibiting nuclear factor-kappa $B$ $(\mathrm{NF}-\kappa \mathrm{B})$ activation. ${ }^{13,15}$

The development of a regimen consisting of a phytochemical with chemopreventive activity combined with celecoxib would be highly desirable for the treatment of cancer, which will reduce the dose of celecoxib ${ }^{16,17}$ and may minimize doserelated cardiovascular side effects. ${ }^{18-20}$ Acetylbritannilactone $(A B L)$, a new active extract isolated from a traditional Chinese medicinal herb Inula britannica $L,{ }^{21}$ is commonly used to treat bronchitis and inflammation in China. Previous studies have shown that $A B L$ exhibit potent anti-inflammatory effects in vascular smooth muscle cells ${ }^{21-23}$ and anticancer activities against colon, breast, ovary, leukemia and prostate cancer

\footnotetext{
${ }^{1}$ Department of Biochemistry and Molecular Biology, Institute of Basic Medicine, Key Laboratory of Neural and Vascular Biology, Ministry of Education, Key Laboratory for Medical Biotechnology of Hebei Province, Hebei Medical University, Shijiazhuang, PR China; ${ }^{2}$ Hebei Provincial Crops Hospital of Chinese People's Armed Police Force, Shijiazhuang, PR China; ${ }^{3}$ Department of Surgery, Fourth Hospital, Hebei Medical University, Shijiazhuang, PR China and ${ }^{4}$ College of Pharmacy, Hebei Medical University, Shijiazhuang, PR China

${ }^{*}$ Corresponding author: M Han, Department of Biochemistry and Molecular Biology, Institute of Basic Medicine, Key Laboratory of Neural and Vascular Biology, Ministry of Education, Key Laboratory for Medical Biotechnology of Hebei Province, Hebei Medical University, No. 361, Zhongshan East Road, Shijiazhuang 050017, PR China. Tel: + 863118626 5563; Fax: + 863118669 6826; E-mail: liubin810103@yahoo.com

Keywords: acetylbritannilactone; apoptosis; breast cancer; cell cycle; cyclooxygenase-2

Abbreviations: ABL, acetylbritannilactone; COX-2, cyclooxygenase-2; ERK, extracellular signal-regulated kinase; JNK, c-Jun NH2-terminal kinase; MAPKs, mitogenactivated protein kinases; NF- $\kappa$ B, nuclear factor-kappa B; NSAIDs, non-steroidal anti-inflammatory drugs; p38, p38 MAP kinase; PARP, poly (ADP-ribose) polymerase; $\mathrm{PGE}_{2}$, Prostaglandin $\mathrm{E}_{2} ;$ siRNA, small interfering RNA

Received 25.4.11; revised 16.6.11; accepted 17.6.11; Edited by A Stephanou
} 
a
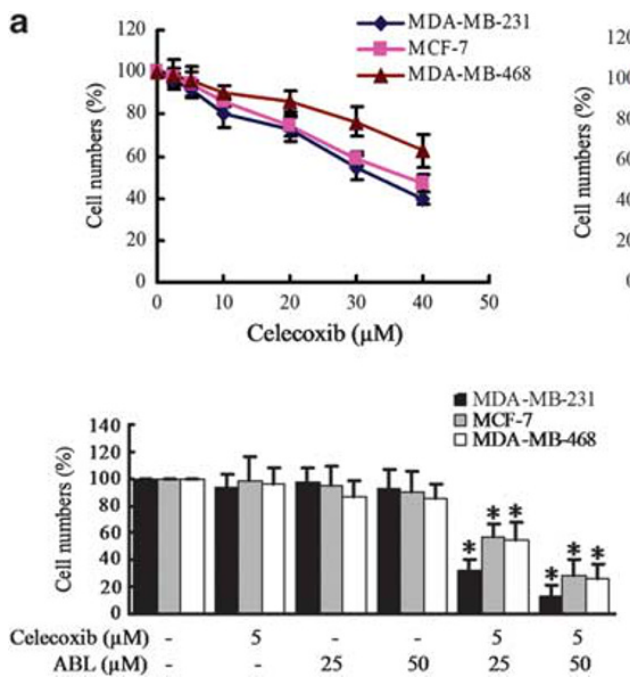
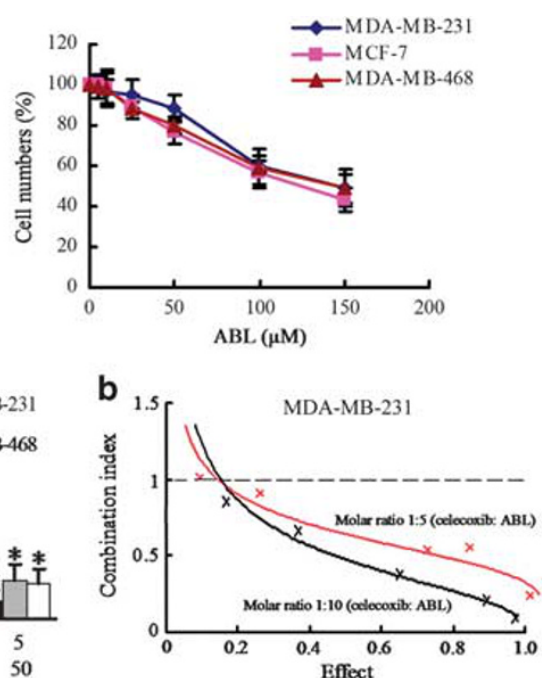

Figure 1 Effects of celecoxib, ABL or their combination on the growth of breast cancer cells. (a) Cells were treated with different doses of celecoxib, ABL or their combination for $48 \mathrm{~h}$, and growth inhibition was measured by cell counting assays. The data were showed as the percentage of vehicle-treated cells. Results were expressed as mean \pm S.E.M. ${ }^{*} P<0.05$, compared with vehicle-treated group. (b) MDA-MB-231 cells were treated with celecoxib $(2.5-10 \mu \mathrm{M})$ plus ABL (12.5-100 $\left.\mu \mathrm{M}\right)$ at a fixed ratio of $1: 5$ or 1:10, and then the $\mathrm{Cl}$ was calculated as described in Materials and Methods

cells. $^{24-27}$ Moreover, additional researches reported that $\mathrm{ABL}$ could activate caspases via the mitochondrial apoptosis pathway, induce $\mathrm{Bcl}-2$ phosphorylation and suppress NF- $\kappa \mathrm{B}$ activation in several types of cell lines. ${ }^{21,25,27}$ Therefore, it is interesting to investigate whether $\mathrm{ABL}$ could be a synergist to help increase the efficacy of other chemopreventive agents in cancer therapy. With the goal of enhancing the chemopreventive effects of celecoxib and reduced the dose in chemotherapy, we evaluated the possibility of the combination treatment of celecoxib and $\mathrm{ABL}$ on human breast cancer in vivo and in vitro.

\section{Results}

The combination treatment of celecoxib and $A B L$ on human breast cancer cell growth. The effects of celecoxib, $\mathrm{ABL}$ and their combination on cell growth were assessed by cell counting assays. Three cell lines (MDA-MB231, MDA-MB-468 and MCF-7) that differ in the COX-2 status (Supplementary Figure S1A) were used to elucidate whether the effects depend on COX-2 expression levels. As shown in Figure 1a, both celecoxib and ABL suppressed cell growth in a dose dependent manner. At the doses tested, neither celecoxib $(5 \mu \mathrm{M})$ nor ABL $(25$ or $50 \mu \mathrm{M})$ elicited the growth-inhibition effect as a single agent, but the combination treatment significantly suppressed cell growth in all three cell lines. Despite the higher basal level of COX-2 expression in MDA-MB-231 cells, a comparable degree of growth inhibition was observed in the other cell lines, which were not found to differ significantly according to COX-2 expression levels. In the presence of $50 \mu \mathrm{M} \mathrm{ABL}, \mathrm{IC}_{50}$ of celecoxib was $4.6 \mu \mathrm{M}$, a 7.5-fold lower dose than the $\mathrm{IC}_{50}(34.4 \mu \mathrm{M})$ of celecoxib alone in MDA-MB-231 cells, suggesting that ABL may enhance the sensitivity of cancer cells to celecoxib. Moreover, there was a dose-response reduction in MDA-MB-231 cell growth caused by the combination treatment (Supplementary Figure S1B).
To do mathematical analysis by the median effect method, MDA-MB-231 cells were treated with both celecoxib and ABL simultaneously at fixed 1: 5 and 1:10 dose ratio for $48 \mathrm{~h}$. Combination index $(\mathrm{Cl})$ values were calculated as described previously. ${ }^{28}$ As shown in Figure $1 \mathrm{~b}$, the $\mathrm{Cl}$ values of the concentration were less than 1 , indicating that $A B L$ synergistically enhances celecoxib-induced growth inhibition in MDAMB-231 cells. Moreover, similar results were obtained in MDA-MB-468 and MCF-7 cell lines (Supplementary Figure $\mathrm{S1C})$. Thus, celecoxib and $\mathrm{ABL}$ may be an effective combination for cell growth inhibition due to their synergistic efficacy.

ABL synergistically enhanced celecoxib-induced apoptosis. To evaluate the mechanism for synergistic cytotoxicity between celecoxib and $A B L$, the extent of apoptosis was assessed using ELISA. In MDA-MB-231 cells, the formation of cytoplasmic histone-associated DNA fragments was observed only when they were exposed to the combination (Figure 2a). DAPI staining assay confirmed the results (Supplementary Figure S2A). However, a very weak effect was seen in MCF-7 cells that expressed no constitutive COX-2 (Figure 2a and Supplementary Figure S2A). Various $\mathrm{ABL}$ doses were tested in the MDA-MB-468 cells, but we were unable to detect any apoptotic enhancement effect of celecoxib $(5 \mu \mathrm{M})$ (Supplementary Figure S2B).

Moreover, apoptosis markers were analyzed by western blot. The combination treatment, instead of individual agents, resulted in marked increased cleavage of PARP in MDA-MB231 cells and, to a minor degree, in MCF-7 cells (Supplementary Figure S2C). Further experiments indicated that the combination treatment increased the active form of cleaved caspase-3 and cleaved caspase- 9 in MDA-MB-231 cells. The absence of caspase- 3 activation in MCF-7 cells ${ }^{29}$ raised the possibility that the combination treatment might induce apoptosis through other caspases or death receptor pathways. 


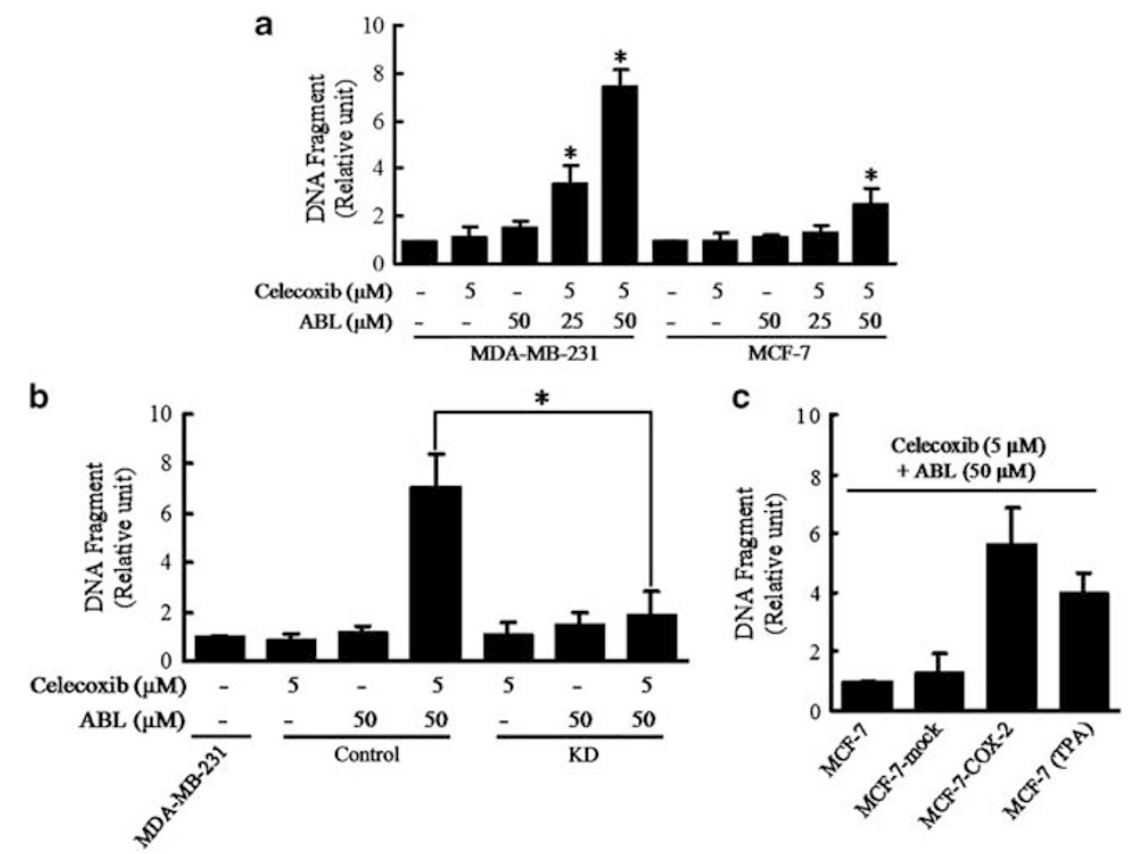

Figure 2 Effects of celecoxib, $A B L$ or their combinations on the apoptosis of breast cancer cells. Cells were treated with celecoxib, ABL or their combination as indicated for $48 \mathrm{~h}$. (a) DNA fragmentation of apoptotic cells was evaluated using a Cell Death Detection ELISA ${ }^{\text {Plus }}$ kit. ${ }^{*} P<0.05$, compared with vehicle-treated cells. (b) Apoptotic cells were evaluated by ELISA in Control and KD cells after the combination treatment. Control, MDA-MB-231 cells transfected with a general negative sequence of siRNA; KD, COX-2 KD MDA-MB-231 cells. (c) MCF-7-mock, MCF-7-COX-2 and TPA (0.1 $\mu$ M)-stimulated MCF-7 cells were treated with combination of celecoxib and ABL for $48 \mathrm{~h}$, and apoptotic cells were evaluated. Results were expressed as mean \pm S.E.M. from at least three independent experiments

The combination-induced apoptosis via a cOX-2-dependent manner. To determine whether the combination-induced apoptosis occurs in a COX-2-dependent route, COX-2 knocked-down cells (KD), which transfected COX-2 siRNA, and its mock control cells (Control) were established from MDA-MB-231 cells. The COX-2 expression was markedly reduced in the $\mathrm{KD}$ compared with MDA-MB-231 cells, whereas we observed no changes in the Control (Supplementary Figure $\mathrm{S} 3 \mathrm{~A}$ ). $\mathrm{PGE}_{2}$ is a primary product of the COX-2 enzyme, which represents COX-2 activity. $\mathrm{PGE}_{2}$ production of the Control cells was comparable to that in MDA-MB-231 cells. However, $\mathrm{PGE}_{2}$ production in the $\mathrm{KD}$ was noted to have decreased to $5 \%$ that observed in MDAMB-231 cells or the Control (Supplementary Figure S3A). Moreover, the combination-induced apoptosis were observed in the Control, but these effects disappeared in the KD (Figure 2b).

Furthermore, additional experiments were performed to determine whether the weak apoptotic induction by combination treatment in MCF-7 cells could be reversed via COX-2 overexpression. For this, we constructed stably transfected MCF-7 cells (MCF-7-COX-2) and mock control cells (MCF-7mock) as previously described. ${ }^{30}$ The MCF-7-COX-2 cells exhibited high expression of COX-2 protein, comparable to that of MDA-MB-231 cells, whereas the MCF-7-mock cells expressed no COX-2 (Supplementary Figure S3B). As shown in Figure 2c, MCF-7-COX-2 cells were more sensitive to combination treatment than MCF-7-mock cells. Moreover, TPA $(0.1 \mu \mathrm{M})$-stimulated MCF-7 cells were treated with the combination and showed that there was additive proapoptotic effect in the cells (Figure 2c). In addition, exogenous $\mathrm{PGE}_{2}$ partially prevented the combination-induced apoptosis in
MDA-MB-231 and MCF-7-COX-2 cells (Supplementary Figure $\mathrm{S} 3 \mathrm{C}$ ), suggesting that these effects at least partially suppress $\mathrm{PGE}_{2}$ generation.

ABL synergistically improved the effects of celecoxib on COX-2 expression and activity. To determine whether the synergistic effects observed in COX-2 expressing cells were related to COX-2 synthesis, expression of COX-2 mRNA and protein was examined in MDA-MB-231 cells. As shown in Figure $3 a$, COX-2 was slightly decreased by the single agent, but was markedly decreased by the combination treatment, although COX-1 was not changed under the same treatment conditions. The combination treatment also notably decreased $\mathrm{PGE}_{2}$ in medium collected from the cells (Figure 3b). Consistent with western blot results, the combination treatment on MDA-MB-231 cells led to a drastic decrease in cytoplasmic COX-2, and COX-1 expression was not affected in immunofluorescence staining analyses (Supplementary Figure S4A).

Moreover, luciferase-reporter construct containing a fragment of COX-2 promoter was transfected into MCF-7 cells, which was used to study regulation of COX-2 transcriptional activity. ${ }^{31,32}$ It showed that $A B L$ potentiated the inhibitory effect of celecoxib on COX-2 activity (Supplementary Figure S4B). In addition, TPA-induced COX-2 expression was also markedly suppressed by the combination treatment (Supplementary Figure S4C).

The combination-induced cell growth inhibition is mediated partly through $\mathbf{G}_{0} / G_{1}$ arrest. As cell growth suppression often occurs as a consequence of cell cycle block, we were interested in knowing whether the effects of 

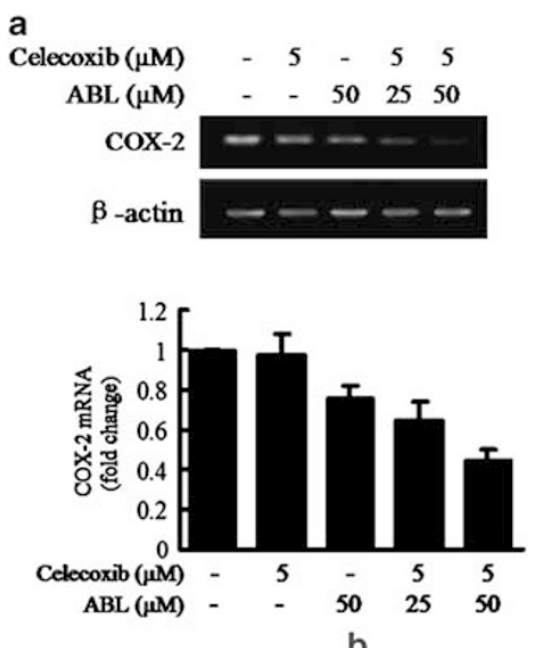
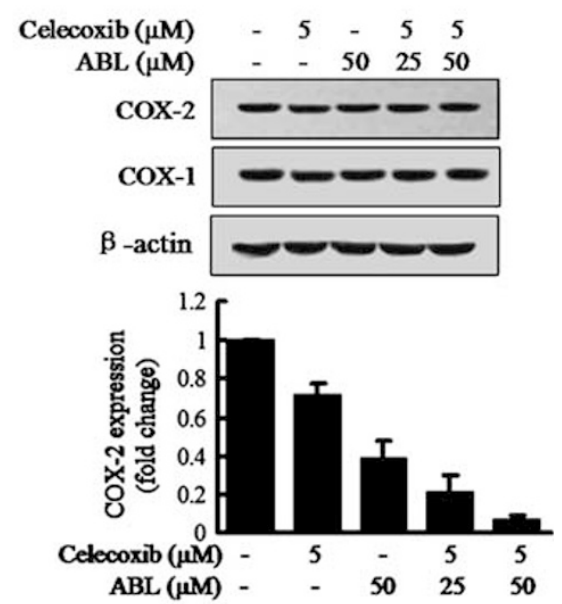

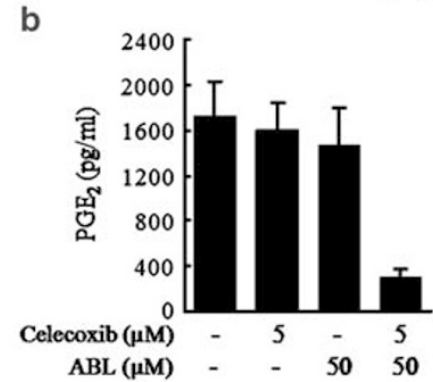

Figure 3 Effects of the combination of celecoxib and ABL on COX-2 expression and activity in MDA-MD-231 cells. (a) MDA-MD-231 cells were treated with celecoxib, ABL or their combination for $24 \mathrm{~h}$ (left) or $48 \mathrm{~h}$ (right). COX-2 mRNA and protein expression was examined using RT-PCR (left) and western blot analysis (right). Quantification of COX-2 mRNA and protein expression normalized to actin levels, were provided at the bottom. (b) MDA-MB-231 cells were treated with the combination for $48 \mathrm{~h}$, and PGE 2 levels in the culture medium were measured using ELISA. Results were expressed as mean \pm S.E.M. from at least three independent experiments

the combination treatment were related to cell cycle regulation. As determined by flow cytometry, the combination of celecoxib and $A B L$ induced a significant inhibition of cell cycle progression from the $G_{0} / G_{1}$ to $S$ phase in both MDA-MB-231 and MCF-7 cells (Figure 4a). Moreover, a marked reduction of cyclinD1 and cyclinE expression was observed when cells were treated with the combination (Figure 4b). CDK2 and CDK6 levels also decreased. Conversely, expression of p21 was significantly induced after the combination treatment. In addition, other tested cell lines treated with celecoxib and $A B L$ also exhibited considerably increased rates of $G_{0} / G_{1}$ phase cells (for Control, KD, MCF-7-COX-2, MCF-7-mock and MDA-MB468 cells, respectively), and supplementation with $\mathrm{PGE}_{2}$ (50-200 pg/ml) did not affect the $\mathrm{G}_{0} / \mathrm{G}_{1}$ block (Supplementary Figure S5A). As p21 has a critical role in mediating $G_{0} / G_{1}$ arrest, we performed experiments to examine whether overexpression of COX-2 would alter its expression. As shown in Supplementary Figure S5B, although the COX-2 protein was induced in MCF-7-COX-2 and TPA-stimulated MCF-7 cells, p21 level was not altered after the combination treatment. Thus, the results suggested that the combinationinduced cell growth inhibition was mediated, at least in part, through $G_{0} / G_{1}$-cell cycle arrest, which was independent of whether the cells expressed COX-2 or not.

The combination-induced cell growth inhibition associated with Akt and p38 signaling. To investigate the common mechanistic basis of apoptosis and cell cycle arrest for the synergistic effects between celecoxib and $A B L$, we determined the changes on the survival signaling pathways represented by Akt, and also the mitogenactivated protein kinases cascades. ${ }^{33}$ Western blot analysis showed that exposure of MCF-7 cells to celecoxib or ABL alone for $2 \mathrm{~h}$ resulted in a slight decrease in Akt and p38 phosphorylation induced by TPA (Figure 5a). However, the combination treatment reduced active Akt and p38 to a greater degree versus either agent alone. In addition, combined exposure of cells to celecoxib and $A B L$ resulted in a modest but discernible decline in the phosphorylation of downstream Akt target GSK-3 $\beta$. In contrast, JNK and ERK1/2 activation were barely affected (Figure $5 \mathrm{a}$ ), suggesting that they were unlikely to be involved in these processes. Focusing on Akt and p38, modulation of these pathways was not cell line specific, as similar results were observed in MDA-MB-231 cells after combination treatment.

To address the question on whether the observed changes in signaling were responsible for the apoptosis, MCF-7 cells were transfected with Ca-Akt or MKK6b vectors. It showed that Ca-Akt or MKK6b protected cells from combinationinduced apoptosis, whereas inhibition of these pathways enhanced pro-apoptotic effects (Figure 5b). Moreover, MCF-7 cells were transfected with COX-2-Luc along with Ca-Akt or MKK6b vectors and it could restore the TPA-induced COX-2 activity after the combination treatment (Supplementary Figure S6A). Further works were addressed to evaluate the ability of celecoxib plus ABL on COX-2 mRNA decay, and similar effects were observed (Supplementary Figure S6B). 
a

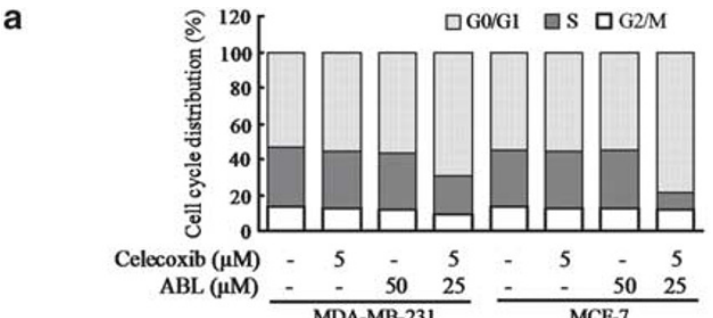

b
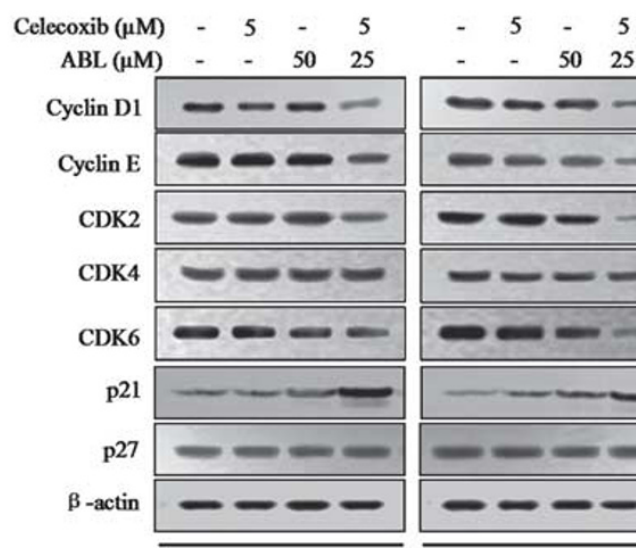

MDA-MB-231
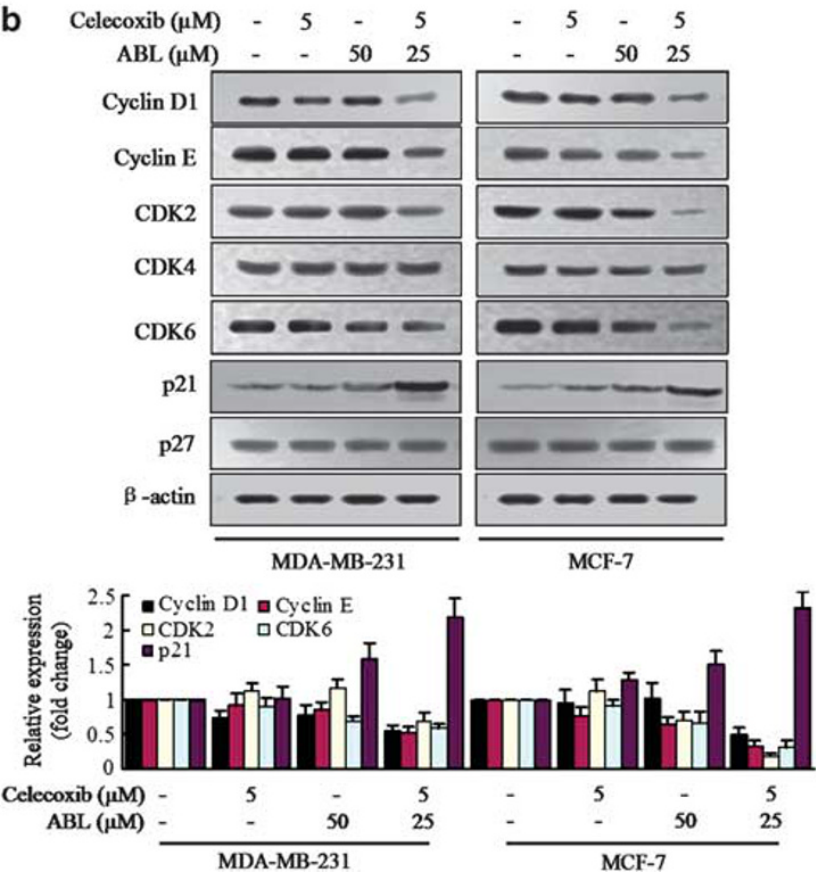

Figure 4 Effects of the combination of celecoxib and $A B L$ on cell cycle progression. (a) MDA-MB-231 and MCF-7 cells were treated with celecoxib, ABL alone or their combination for $24 \mathrm{~h}$. Cell cycle distributions were determined by flow cytometry analysis. The bar graph reflected the percentage of cells in $G_{0} / G_{1}, S, G_{2} / M$ phase of cell cycle. (b) Cells were treated with the combination for $24 \mathrm{~h}$ and subjected to western blot with specific antibodies directed against cyclin D1, cyclin E, CDK-2, CDK-4, CDK-6, p21 and p27. Densitometric analyses of western blot, normalized to actin levels, were provided. Each column represents the mean \pm S.E.M.

These results indicated that the combination treatment modulated the activation of Akt and p38, beyond the primary effect on COX-2.

Subsequently, the roles of the above signal pathways were determined in cell cycle regulation. As shown in Figure $5 \mathrm{c}$, activation of the Akt or p38 pathway significantly decreased cell number in $G_{0} / G_{1}$ phase, whereas inhibition of these pathways enhanced cell $G_{0} / G_{1}$ cycle arrest by the combination treatment. Thus, it indicated that the synergism reflected inhibition of Akt and p38 that occurred upstream of combination-initiated cell cycle arrest.

Therapeutic effects of combination treatment against MDA-MB-231 xenograft model. As the combination treatment of celecoxib and $A B L$ was more effective than the individual agents in vitro, effects of them on tumor development were examined in athymic nude mice. As shown in Figure 6a, treatment with celecoxib $(5 \mathrm{mg} / \mathrm{kg})$ or $\mathrm{ABL}(15 \mathrm{mg} / \mathrm{kg})$ had weak inhibitory effect on MDA-MB-231 tumor growth, whereas the combination treatment significantly reduced tumor volume by $50 \% \quad(P<0.05)$ after 30 days. All the groups were well tolerated without obvious signs of drug-related toxicity throughout the course of this study.

Furthermore, the COX-2 expression at tumor specimens was measured. Compared with the individual use of one agent, it clearly indicated that tumor specimens from celecoxib plus $A B L$-treated mice stained more weakly positive COX-2 expression (Supplementary Figure S7A). Similar results were also determined by western blot analysis in protein extracted from the tumor tissue (Figure 6b). The findings from ELISA assay for serum $\mathrm{PGE}_{2}$ level showed a significant decrease in mice that received the combination treatment (Supplementary Figure S7B). Further, in-depth analysis indicated a positive correlation between the serum $\mathrm{PGE}_{2}$ level and tumor COX-2 expression (Pearson's correlation coefficient, 0.900; $P<0.001$, two-tailed) (Figure $6 \mathrm{c}$ ). Moreover, augmented expression of p21 and marked increased cleavage of PARP (Figure 6b) was evident in the mice that received the combination treatment, confirming in vitro data.

We finally evaluated whether the effects of the combination treatment on signal transduction could be also observed in vivo. As shown in Figure 6b, although the inhibitory effects exerted by the individual agents were mild, total and phosphorylated Akt and p38 were downregulated among the mice that received the combination treatment.

\section{Discussion}

In this study, we found that the phytochemical ABL synergistically augmented the growth-inhibitory effects of celecoxib on breast cancer cells via COX-2-dependent apoptosis and COX-2-independent cell cycle regulation involving Akt and p38 pathways. Moreover, the therapeutic potential of this combination was shown in its superior activity in suppressing the xenograft tumor growth comparing with that of individual agents.

The combinatorial strategies in cancer therapies can provide more synergistic tumor inhibition and less systemic toxicity than each of the monotherapeutic regimens alone. ${ }^{34-36}$ Current results showed that $A B L$ was useful to increase the anticancer activity of celecoxib and reduce its dose. Low dose of celecoxib $(5 \mu \mathrm{M})$ plus $\mathrm{ABL}(50 \mu \mathrm{M})$ was sufficient to suppress breast cancer cell growth in vitro. This effect was more than that exerted by a higher dose of celecoxib $(>40 \mu \mathrm{M})$ when administered alone. The dose of celecoxib using here was lower than clinically achievable plasma levels. ${ }^{37}$ It might be clinically useful, because it could be achieved in the patients who receive the standard dose of celecoxib to cure the neoplastic disease and also avoids the related cardiovascular risk. ${ }^{18-20}$ Moreover, we found that $A B L$ primarily affects the sensitization of COX-2expressing cells to celecoxib and does not tend to confer this sensitivity to COX-2-nonexpressing cells. Thus, it would be reasonable to suppose that $\mathrm{ABL}$-augmented celecoxibinduced apoptosis occurs via a COX-2-dependent mechanism. 
a

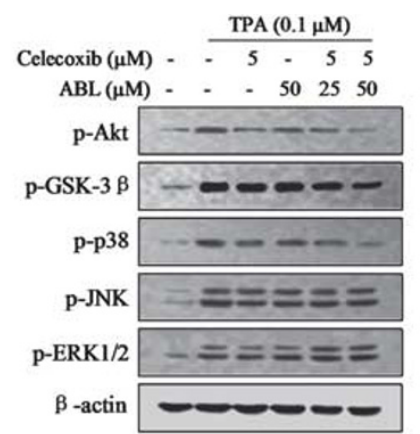

b

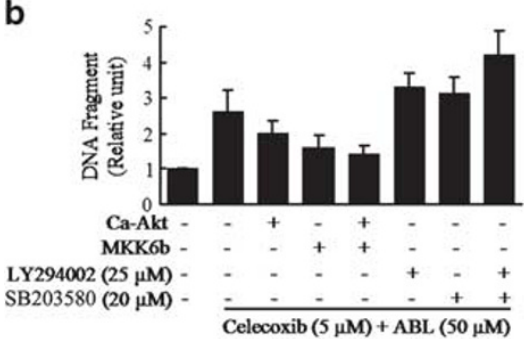

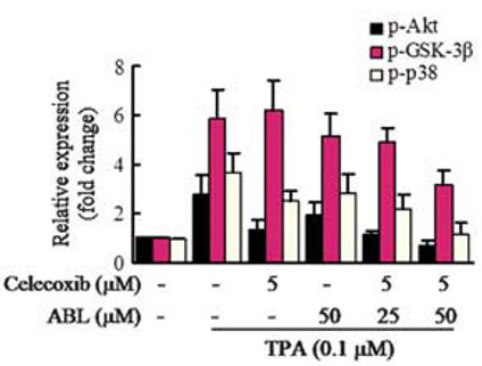

C

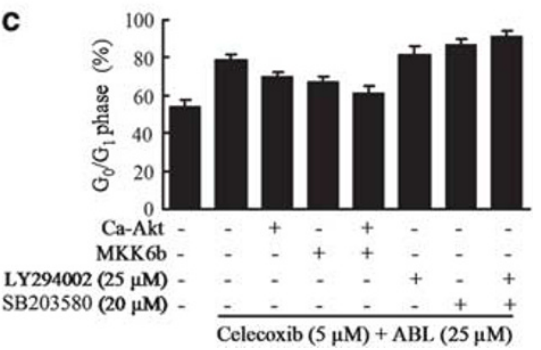

Figure 5 Effects of the combination of celecoxib and ABL on signaling pathways in MCF-7 cells. (a) MCF-7 cells were cotreated with TPA and the combination for $2 \mathrm{~h}$, then cell lysates were analyzed by western blot for p-Akt, p-GSK-3 $\beta$, p-p38, p-JNK and p-ERK1/2. Densitometric analyses of western blot, normalized to actin levels, were provided. (b) MCF-7 cells were transfected with the Ca-Akt or MKK6b vector and then treated with TAP and the combination in the presence of Akt inhibitor (LY294002) or p38 inhibitor (SB203580) for $48 \mathrm{~h}$ as indicated. The percentage of apoptotic cells was determined by ELISA. (c) The transfected cells were treated with the combination in the presence of LY294002 or SB203580 for $24 \mathrm{~h}$. Cell cycle distribution was analyzed by flow cytometry. Results were expressed as mean \pm S.E.M.
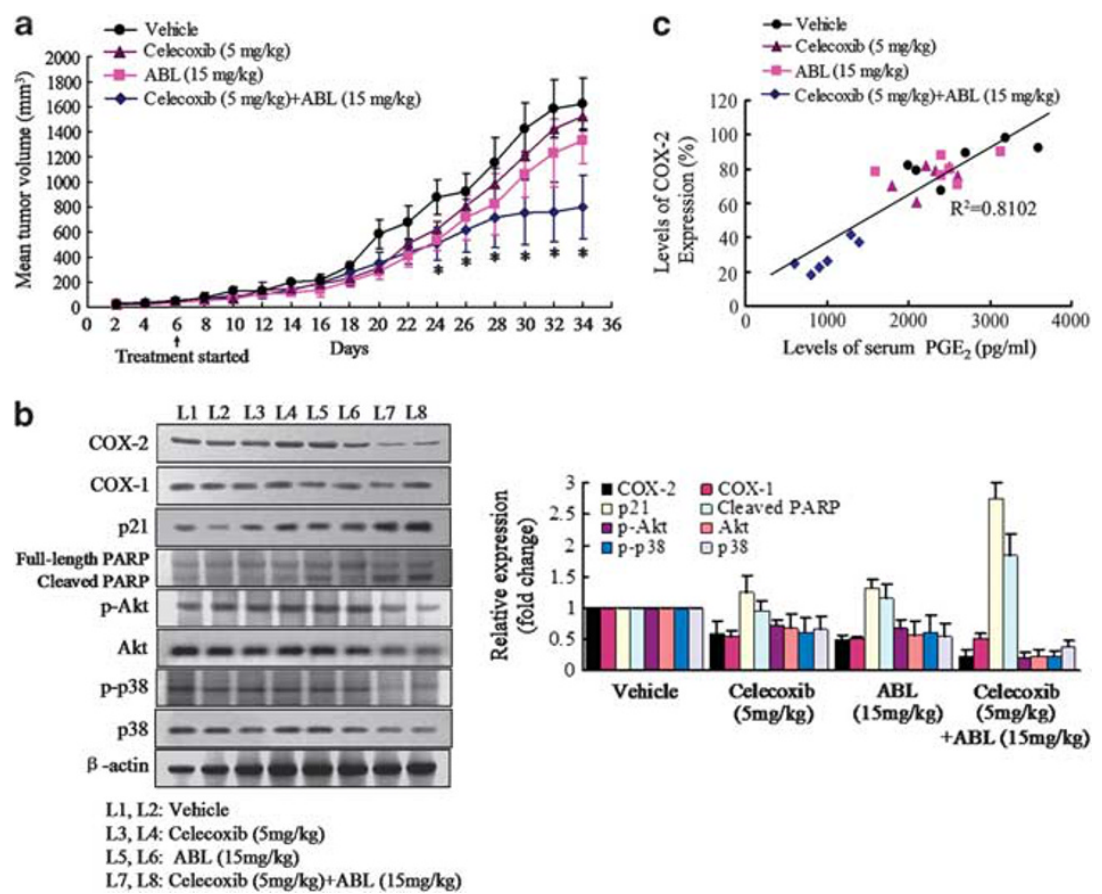

Figure 6 Effects of celecoxib and ABL individually and in combination on MDA-MB-231 Xenograft model. Female nude mice bearing MDA-MB-231 tumors were treated with vehicle, celecoxib $(5 \mathrm{mg} / \mathrm{kg}$ ) and $\mathrm{ABL}(15 \mathrm{mg} / \mathrm{kg})$ individually, or in combination. (a) Tumors were measured with calipers on alternate days. Points, mean tumor volume in each experimental group containing six mice. ${ }^{*} P<0.01$ versus vehicle group. (b) The expression of COX-2, COX-1, p21, PARP, p-Akt, Akt, p-p38 and p38 were determined by western blot analysis in protein extracted from the tumor specimens. Total protein samples from two mice for each group representing control and experiment were used for western blot. Densitometric analyses of western blot (average of two samples) were provided. Each column represents the mean \pm S.E.M. (c) A strong positive correlation (data shown were obtained from Supplementary Figure S7A and B) between serum PGE $\mathrm{P}_{2}$ level and COX-2 expression in xenograft specimens (Pearson's correlation coefficient, 0.900; $P<0.001$, two-tailed) 
Furthermore, we observed that only the combination treatment decreased COX-2 expression, activity and notably reduced $\mathrm{PGE}_{2}$ production in COX-2-overexpressing cells. It can be inferred that the synergistic effects may be not only mediated by COX-2 protein itself or by substrates or enzymes in the upstream regions of this protein, but also by its end product. In addition, the combination treatment has no effect on COX-1 expression in vitro and in vivo experiment, it is reasonable to assume that it may be avoid of the side effects associated with the suppression of COX-1-derived prostanoids.

Many studies have showed that COX-2 might be associated with drug resistance. Several treatment, such as paclitaxel, ${ }^{38}$ mitomycin $\mathrm{C}^{39}$ and radiation therapy, ${ }^{40}$ induced COX-2 overexpression, resulting in some adverse effects associated with inflammation, angiogenesis, invasion and metastasis. ${ }^{1,10,41}$ Therefore, downregulation of COX-2 expression might provide effective approaches to treat cancer patients who were sufferings drug resistance. Previous studies showed that the prototype tumor promoter TPA induced COX-2 expression in MCF-7 cells by activating transcription factors such as NF- $\kappa \mathrm{B}$ and activator protein-1 (AP-1). ${ }^{31,32}$ In the present study, we used TAP-induced MCF-7 cells to simulate the condition of drug resistance related to COX-2 overexpression. Surprisingly, the combination treatment suppressed COX-2 activation and induced significant apoptosis in TPA-stimulated MCF-7 cells, suggesting that the therapeutic regimen might be helpful in drug resistance related to COX-2 expression.

As cell growth inhibition often occurs as a result of cell cycle arrest, we determined the second mechanism underlying the synergistic effects. Previous studies have shown that treatment with low dose of celecoxib $(<20 \mu \mathrm{M})$ alone for a short time $(<72 \mathrm{~h})$ may not affect cell viability and cell cycle distribution. ${ }^{14,42}$ Conversely, we found that the combination treatment of celecoxib $(5 \mu \mathrm{M})$ and $\mathrm{ABL}(50 \mu \mathrm{M})$ for $24 \mathrm{~h}$ induced $\mathrm{G}_{0} / \mathrm{G}_{1}$ arrest in all the tested cells. The incomplete protection by addition of $\mathrm{PGE}_{2}$ or COX-2 overexpression, and the fact that overexpression or depletion of COX-2 failed to alter the cell cycle indicated the existence of COX-2independent mechanism. Moreover, the combinationmediated cell cycle arrest could be caused by the suppression of cyclinD1, cyclinE, CDK2 and CDK6 activities, especially the upregulation of p21, which were significant and consistent with the earlier studies that increased p21 expression were associated with inactive of CDKs/cyclin complexes and induced of $\mathrm{G}_{0} / \mathrm{G}_{1}$ arrest. ${ }^{43,44}$

Another important observation in this study was that celecoxib combined with $\mathrm{ABL}$ suppressed phosphorylated Akt and p38. Activated Akt and p38 targeted multiple factors involving in cell proliferation, survival and also mediating cell cycle alteration and apoptosis. Recently, many studies have demonstrated that the cytotoxic compounds could suppress cancer cell growth through these signaling pathways. Flavopiridol could interfere with Akt and p38 and enhanced cell apoptosis. ${ }^{45}$ Furthermore, Akt and p38 were identified as key signaling responsible for paclitaxel in metastatic breast cancer. ${ }^{46}$ In the presence of $\mathrm{ABL}$, celecoxib dramatically downregulated Akt and p38 pathways so as to facilitate apoptosis and cell cycle arrest. Thus, we hypothesize that Akt and p38 signaling represent a therapeutically relevant target for $\mathrm{ABL}$ to sensitize these cells to celecoxib via two potential mechanisms. First, the suppression of these pathways by the combination treatment would lead to the inhibition of COX-2 expression and activity, which triggered the apoptosis regulators to facilitate apoptosis. Second, ABL interacted synergistically with celecoxib, which could block Akt and p38 activation. Thereby, this process influenced the cell cycle regulator proteins and induced $\mathrm{G}_{0} / \mathrm{G}_{1}$ phase cell arrest.

In summary, $A B L$ synergistically enhanced the activity of celecoxib on breast cancer growth inhibition through COX-2dependent and COX-2-independent mechanisms. Findings from this study concluded that the combination treatment of celecoxib and $\mathrm{ABL}$ could improve the chemopreventive efficacy certainly in breast cancer and help to explain the anticancer properties of these chemopreventive agents.

\section{Materials and Methods}

Reagents, antibodies and plasmids. Celecoxib was provided by LKT Laboratories, Inc. (Minneapolis, MN, USA). Silica gel column chromatography was used to isolate $\mathrm{ABL}$ from Inula britannica $L$ grown in Shan-xi Province in China. The effects of $A B L$ and celecoxib in our experiments were compared with the same concentration of dimethyl sulfoxide (DMSO) as vehicle. The antibodies specific for Poly (ADP-ribose) polymerase (PARP), phospho-c-Jun $\mathrm{NH}_{2}$-terminal kinase (p-JNK), phospho-p38 MAP kinase (p-p38) and p38 were obtained from Cell Signaling Technology (Beverly, MA, USA). Antibodies against COX-1, COX-2, $\beta$-actin, caspase-3, caspase-9, phospho-extracellular signal-regulated kinase1/2 (p-ERK1/2), phospho-Akt (p-Akt), Akt, phospho-GSK-3 $\beta$ (p-GSK-3 $\beta$ ), the secondary antibodies, small interfering RNA (siRNA) specific for human COX-2 mRNA and control siRNA were from Santa Cruz Biotechnology (Santa Cruz, CA, USA). Unless otherwise indicated, all other reagents used in this study were obtained from Sigma Chemicals (St. Louis, MO, USA). Constitutively active p38 construct MKK6b was kindly provided by Dr. Han (The Scripps Research Institute, CA, USA). Constitutively active Akt construct Ca-Akt was gifts from Dr. Woodgett (Ontario Cancer Institute, Toronto, Canada).

Cell lines and culture conditions. The human breast cancer cell lines (MDA-MB-231, MDA-MB-468 and MCF-7) were from American Type Culture Collection (Manassas, VA, USA). Cells were grown in a $5 \% \mathrm{CO}_{2}$ atmosphere at $37^{\circ} \mathrm{C}$ in DMEM (Invitrogen, Carlsbad, CA, USA) supplemented with $10 \%$ fetal bovine serum. All treatments were carried out on cells at $60-80 \%$ confluence.

Cell growth inhibition assays. The cells were plated in duplicate in 12-well plates. After $24 \mathrm{~h}$, celecoxib, ABL and their combination were added at the selected doses to the culture medium. The number of viable cells was determined by a Coulter counter $48 \mathrm{~h}$ later. We calculated the $\mathrm{IC}_{50}$ by using a standard sigmoidal Emax model. To determine whether the combined effects were synergistic, the cells were treated with the combination of the indicated doses of celecoxib and ABL for $48 \mathrm{~h}$ and the $\mathrm{Cl}$ was determined using the method of Chou and Talalay through the commercial software package Calcusyn (Biosoft, Cambridge, UK). ${ }^{28}$

Apoptosis assays. Apoptosis was determined by two independent methods. ${ }^{47}$ For fluorescence microscopy, cells were treated with agents for $48 \mathrm{~h}$. Apoptotic cells were detected by nuclear morphologic changes using DAPI staining. Cells were harvested, washed with PBS and fixed in $70 \%$ ethanol for $30 \mathrm{~min}$. The fixed cells were placed on slides and stained with $1 \mathrm{mg} / \mathrm{ml}$ DAPI for $15 \mathrm{~min}$. Excess dye was removed and the monolayer was thoroughly washed with PBS. The stained nuclei were observed via a fluorescence microscope.

For ELISA, the cells seeded in 96-well plates were treated with agents for $48 \mathrm{~h}$. The induction of apoptosis by the agents was evaluated with a Cell Death Detection ELISA ${ }^{\text {Plus }}$ kit (Roche Diagnostics, Mannheim, Germany) according to the manufacturer's instruction. Measurements were made using an ELISA reader at $405 \mathrm{~nm}$ and the results were calculated as the ratio of the absorbance of the agentstreated cells/absorbance of vehicle-treated cells.

Cell cycle analysis. Cells were treated with agents at selected doses and harvested by trypsinization, washed with PBS, fixed with ethanol, incubated for 
5 min with $0.5 \%$ Triton X-100 and stained with propidium iodide in PBS containing $25 \mu \mathrm{g} / \mathrm{ml}$ RNase. Stained cells were analyzed by flow cytometry (Becton Dickinson, San Jose, CA, USA).

Immunohistochemistry. Paraffin-embedded tumor specimens were subjected to immunohistochemistry according to the antibody protocol. Specimens were developed using secondary antibodies linked to HRP and DBA as a substrate.

Immunofluorescence microscopy. Cells were treated with agents at selected doses for $48 \mathrm{~h}$, then fixed on coverslips with $4 \%$ paraformaldehyde, permeabilized with $0.2 \%$ Triton X-100 and blocked in $5 \%$ goat serum, and then incubated overnight at $4{ }^{\circ} \mathrm{C}$ with antibodies, followed by incubation with FITCconjugated secondary antibody for $1 \mathrm{~h}$ at room temperature in the dark. Nuclear counterstaining was performed with $\mathrm{PI}$ for $10 \mathrm{~min}$ at room temperature. Fluorescence was visualized under a fluorescence microscope (Olympus, Tokyo, Japan).

Reverse transcription-PCR (RT-PCR). Total RNA was isolated from cells using TRIzol Reagent (Invitrogen) and equal amounts were reverse transcribed into cDNA using the oligo dT primer, then the cDNAs were used as DNA templates for PCR. Primers used for COX-2 were $5^{\prime}$-TTCAAATGAGATTGTGGGAAAAT- $3^{\prime}$ and $5^{\prime}$-AGATCATCTCTGCCTGAGTATCTT- $3^{\prime}$. $\beta$-actin was used to ensure equal loading. The PCR products were separated on $1 \%$ agarose gel and visualized by ethidium bromide.

Western blot analysis. Equal amounts of proteins were subjected to SDSPAGE analysis and subsequent immunoblotting was carried out with specific antibodies. Levels of immunocomplexes for $\beta$-actin were used as an internal standard for equal loading. All experiments were repeated at least thrice and yielded similar results.

$\mathrm{PGE}_{\mathbf{2}}$ production. $\mathrm{PGE}_{2}$ from the collected cell medium or the animal serum was determined by a commercially available $P_{G_{2}}$-specific ELISA (R\&D Biosystems, Abingdon, UK) according to manufacturer's instructions.

Transfection of siRNA. siRNAs specific for COX-2 (COX-2 siRNA) and control siRNA were transfected into cells using transfection reagent Lipofectamine 2000 (Invitrogen). RT-PCR and western blot were used to confirm adequate silencing of the COX-2 genes at $48 \mathrm{~h}$.

Transfection of cells. Cells were transfected either with empty vector or the same vector containing a cDNA encoding constitutively active p38 construct MKK6b, constitutively active Akt construct Ca-Akt.

The stably transfected cells were conducted using COX-2 expression plasmid (full-length human COX-2 cDNA cloned in pcDNA3 vector) and G418 geneticin (Invitrogen) as described previously. ${ }^{30}$ Individual drug-resistant colonies were isolated and expanded. The selected cells with successful overexpression of COX-2 protein were used for subsequent experiments.

Luciferase assays. The COX-2 luciferase reporter construct (COX-2-Luc) $(-1432 /+59)$, containing the wild-type COX-2 promoter fragment, was prepared and cloned into the $\mathrm{pGL} 3$ basic vector. Cells were plated in 24-well plates. Transient transfections were performed using the transfection reagent Lipofectamine 2000. Each well was co-transfected with COX-2-Luc plasmid and the internal control plasmid renilla luciferase (pRL-TK). The cells were harvested after the indicated times of treatment, and the activities of both firefly luciferase and pRL-TK luciferase were measured.

Xenograft model. The animal study was performed via a protocol approved by the governmental committee for animal research. Female BALB/c nude mice (4-5 weeks old) were injected s.c. with MDA-MB-231 cells $\left(6 \times 10^{6}\right)$ per mice at both flanks and randomly assigned to treatment groups (six mice per group). After 6 days, when a small vascularized tumor $\left(50-100 \mathrm{~mm}^{3}\right)$ had developed, control group was treated with vehicle (10\% DMSO, $10 \%$ ethanol in water) daily by i.p. injection. The celecoxib group $(5 \mathrm{mg} / \mathrm{kg}$ ) was treated daily by oral administration as described previously. ${ }^{10} \mathrm{ABL}$ group ( $15 \mathrm{mg} / \mathrm{kg}$ ) was given daily by i.p. injection. The group treated with the combination was dosed in the same manner as both single-agent groups. Mice were weighed at least twice a week and the tumor size was measured every other day using calipers and their volumes were calculated according to a standard formula: width ${ }^{2} \times$ length/2. Mice were killed after 36 days of treatment and the tumor specimens were removed for immunohistochemistry and western blot analysis.

Statistical analysis. All of the experiments were repeated a minimum of three times. Data were presented as mean \pm S.E.M. Statistical analyses of the differences between the groups were determined with the two-sided unpaired Student's t-test using SPSS software (SPSS, Inc., Chicago, IL, USA), and a value of $P<0.05$ was considered as significant.

\section{Conflict of Interest}

The authors declare no conflict of interest.

Acknowledgements. This work was supported by a grant from the National Natural Science Foundation of PR China (No. 30973820) and a grant from the Hebei Province Natural Science Foundation of PR China (No. C2011206163 and C2011206146).

1. Howe LR, Subbaramaiah K, Brown AM, Dannenberg AJ. Cyclooxygenase-2: a target for the prevention and treatment of breast cancer. Endocr Relat Cancer 2001; 8: 97-114.

2. Bos PD, Zhang XH, Nadal C, Shu W, Gomis RR, Nguyen DX et al. Genes that mediate breast cancer metastasis to the brain. Nature 2009; 459: 1005-1009.

3. Hu M, Peluffo G, Chen H, Gelman R, Schnitt S, Polyak K. Role of COX-2 in epithelialstromal cell interactions and progression of ductal carcinoma in situ of the breast. Proc Natl Acad Sci USA 2009; 106: 3372-3377.

4. Harris RE, Chlebowski RT, Jackson RD, Frid DJ, Ascenseo JL, Anderson G et al. Breast cancer and nonsteroidal anti-inflammatory drugs: prospective results from the Women's Health Initiative. Cancer Res 2003; 63: 6096-6101.

5. Terry MB, Gammon MD, Zhang FF, Tawfik H, Teitelbaum SL, Britton JA et al. Association of frequency and duration of aspirin use and hormone receptor status with breast cancer risk. JAMA 2004; 291: 2433-2440.

6. Hinz B, Brune K. Cyclooxygenase-2-10 years later. J Pharmacol Exp Ther 2002; 300: 367-375.

7. Ulrich CM, Bigler J, Potter JD. Non-steroidal anti-inflammatory drugs for cancer prevention: promise, perils and pharmacogenetics. Nat Rev Cancer 2006; 6: 130-140.

8. Chan FK, Hung LC, Suen BY, Wu JC, Lee KC, Leung VK et al. Celecoxib versus diclofenac and omeprazole in reducing the risk of recurrent ulcer bleeding in patients with arthritis. N Engl J Med 2002; 347: 2104-2110.

9. Harris RE, Alshafie GA, Abou-Issa H, Seibert K. Chemoprevention of breast cancer in rats by celecoxib, a cyclooxygenase 2 inhibitor. Cancer Res 2000; 60: 2101-2103.

10. Basu GD, Pathangey LB, Tinder TL, Lagioia M, Gendler SJ, Mukherjee P. Cyclooxygenase-2 inhibitor induces apoptosis in breast cancer cells in an in vivo model of spontaneous metastatic breast cancer. Mol Cancer Res 2004; 2: 632-642.

11. Grosch S, Maier TJ, Schiffmann S, Geisslinger G. Cyclooxygenase-2 (COX-2)independent anticarcinogenic effects of selective COX-2 inhibitors. J Natl Cancer Inst 2006; 98: 736-747.

12. Kang HK, Lee E, Pyo H, Lim SJ. Cyclooxygenase-independent down-regulation of multidrug resistance-associated protein-1 expression by celecoxib in human lung cancer cells. Mol Cancer Ther 2005; 4: 1358-1363.

13. Shishodia S, Koul D, Aggarwal BB. Cyclooxygenase (COX)-2 inhibitor celecoxib abrogates TNF-induced NF-kappa B activation through inhibition of activation of I kappa B alpha kinase and Akt in human non-small cell lung carcinoma: correlation with suppression of COX-2 synthesis. J Immunol 2004; 173: 2011-2022.

14. Grosch S, Tegeder I, Niederberger E, Brautigam L, Geisslinger G. COX-2 independent induction of cell cycle arrest and apoptosis in colon cancer cells by the selective COX-2 inhibitor celecoxib. FASEB J 2001; 15: 2742-2744.

15. Kim HJ, Hawke N, Baldwin AS. NF-kappaB and IKK as therapeutic targets in cancer. Cell Death Differ 2006; 13: 738-747.

16. Lev-Ari S, Strier L, Kazanov D, Madar-Shapiro L, Dvory-Sobol H, Pinchuk I et al. Celecoxib and curcumin synergistically inhibit the growth of colorectal cancer cells. Clin Cancer Res 2005; $11:$ 6738-6744.

17. Bhatt RS, Merchan J, Parker R, Wu HK, Zhang L, Seery V et al. A phase 2 pilot trial of lowdose, continuous infusion, or 'metronomic' paclitaxel and oral celecoxib in patients with metastatic melanoma. Cancer 2010; 116: 1751-1756.

18. Dogne JM, Hanson J, Supuran C, Pratico D. Coxibs and cardiovascular side-effects: from light to shadow. Curr Pharm Des 2006; 12: 971-975.

19. Solomon SD, Pfeffer MA, McMurray JJ, Fowler R, Finn P, Levin B et al. Effect of celecoxib on cardiovascular events and blood pressure in two trials for the prevention of colorectal adenomas. Circulation 2006; 114: 1028-1035. 
20. Solomon SD, McMurray JJ, Pfeffer MA, Wittes J, Fowler R, Finn P et al. Cardiovascular risk associated with celecoxib in a clinical trial for colorectal adenoma prevention. $\mathrm{N}$ Engl $\mathrm{J} \mathrm{Med}$ 2005; 352: 1071-1080.

21. Liu B, Han M, Wen JK. Acetylbritannilactone Inhibits Neointimal Hyperplasia after Balloon Injury of Rat Artery by Suppressing Nuclear Factor-\{kappa\}B Activation. J Pharmacol Exp Ther 2008; 324: 292-298.

22. Liu YP, Wen JK, Zheng B, Zhang DQ, Han M. Acetylbritannilactone suppresses lipopolysaccharide-induced vascular smooth muscle cell inflammatory response. Eur $J$ Pharmacol 2007; 577: 28-34.

23. Liu B, Han M, Sun RH, Wang JJ, Liu YP, Wen JK. Acetylbritannilactone induces G(1) arrest and apoptosis in vascular smooth muscle cells. Int J Cardiol 2011; 149: 30-38.

24. Bai N, Lai CS, He K, Zhou Z, Zhang L, Quan Z et al. Sesquiterpene lactones from Inula britannica and their cytotoxic and apoptotic effects on human cancer cell lines. J Nat Prod 2006; 69: 531-535.

25. Rafi MM, Bai NS, Chi Tang H, Rosen RT, White E, Perez D et al. A sesquiterpenelactone from Inula britannica induces anti-tumor effects dependent on Bcl-2 phosphorylation Anticancer Res 2005; 25: 313-318.

26. Park EJ, Kim J. Cytotoxic sesquiterpene lactones from Inula britannica. Planta Med 1998; 64: 752-754.

27. Pan MH, Chiou YS, Cheng AC, Bai N, Lo CY, Tan D et al. Involvement of MAPK, Bcl-2 family, cytochrome $\mathrm{C}$, and caspases in induction of apoptosis by 1,6-0,0diacetylbritannilactone in human leukemia cells. Mol Nutr Food Res 2007; 51: 229-238.

28. Chou TC, Talalay P. Quantitative analysis of dose-effect relationships: the combined effects of multiple drugs or enzyme inhibitors. Adv Enzyme Regul 1984; 22: 27-55.

29. Janicke RU, Sprengart ML, Wati MR, Porter AG. Caspase-3 is required for DNA fragmentation and morphological changes associated with apoptosis. J Biol Chem 1998; 273: 9357-9360.

30. Mehar A, Macanas-Pirard P, Mizokami A, Takahashi Y, Kass GE, Coley HM. The effects of cyclooxygenase-2 expression in prostate cancer cells: modulation of response to cytotoxic agents. J Pharmacol Exp Ther 2008; 324: 1181-1187.

31. Degner SC, Kemp MQ, Bowden GT, Romagnolo DF. Conjugated linoleic acid attenuates cyclooxygenase-2 transcriptional activity via an anti-AP-1 mechanism in MCF-7 breast cancer cells. J Nutr 2006; 136: 421-427.

32. Liu XH, Rose DP. Differential expression and regulation of cyclooxygenase- 1 and -2 in two human breast cancer cell lines. Cancer Res 1996; 56: 5125-5127.

33. Chang YJ, Wu MS, Lin JT, Chen CC. Helicobacter pylori-Induced invasion and angiogenesis of gastric cells is mediated by cyclooxygenase-2 induction through TLR2 TLR9 and promoter regulation. J Immunol 2005; 175: 8242-8252.

34. Falandry C, Canney PA, Freyer G, Dirix LY. Role of combination therapy with aromatase and cyclooxygenase-2 inhibitors in patients with metastatic breast cancer. Ann Oncol 2009; 20: 615-620.

35. Canney PA, Machin MA, Curto J. A feasibility study of the efficacy and tolerability of the combination of Exemestane with the COX-2 inhibitor Celecoxib in post-menopausal patients with advanced breast cancer. Eur J Cancer 2006; 42: 2751-2756.
36. Mustafa A, Kruger WD. Suppression of tumor formation by a cyclooxygenase-2 inhibitor and a peroxisome proliferator-activated receptor gamma agonist in an in vivo mouse model of spontaneous breast cancer. Clin Cancer Res 2008; 14: 4935-4942.

37. Davies NM, McLachlan AJ, Day RO, Williams KM. Clinical pharmacokinetics and pharmacodynamics of celecoxib: a selective cyclo-oxygenase-2 inhibitor. Clin Pharmacokinet 2000; 38: 225-242.

38. Cassidy PB, Moos PJ, Kelly RC, Fitzpatrick FA. Cyclooxygenase-2 induction by paclitaxel, docetaxel, and taxane analogues in human monocytes and murine macrophages: structure-activity relationships and their implications. Clin Cancer Res 2002; 8: 846-855.

39. Hsueh CT, Chiu CF, Kelsen DP, Schwartz GK. Selective inhibition of cyclooxygenase-2 enhances mitomycin-C-induced apoptosis. Cancer Chemother Pharmacol 2000; 45 389-396.

40. Flockhart RJ, Diffey BL, Farr PM, Lloyd J, Reynolds NJ. NFAT regulates induction of COX2 and apoptosis of keratinocytes in response to ultraviolet radiation exposure. FASEB J 2008; 22: 4218-4227.

41. Chang SH, Liu $\mathrm{CH}$, Conway R, Han DK, Nithipatikom K, Trifan OC et al. Role of prostaglandin E2-dependent angiogenic switch in cyclooxygenase 2-induced breast cancer progression. Proc Natl Acad Sci USA 2004; 101: 591-596.

42. Bock JM, Menon SG, Sinclair LL, Bedford NS, Goswami PC, Domann FE et al. Celecoxib toxicity is cell cycle phase specific. Cancer Res 2007; 67: 3801-3808.

43. Abbas T, Dutta A. p21 in cancer: intricate networks and multiple activities. Nat Rev Cancer 2009; 9: 400-414.

44. Weiss RH. p21Waf1/Cip1 as a therapeutic target in breast and other cancers. Cancer Cell 2003; 4: 425-429.

45. Takada Y, Sethi G, Sung B, Aggarwal BB. Flavopiridol suppresses tumor necrosis factorinduced activation of activator protein-1, c-Jun N-terminal kinase, p38 mitogen-activated protein kinase (MAPK), p44/p42 MAPK, and Akt, inhibits expression of antiapoptotic gene products, and enhances apoptosis through cytochrome c release and caspase activation in human myeloid cells. Mol Pharmacol 2008; 73: 1549-1557.

46. Sunters A, Madureira PA, Pomeranz KM, Aubert M, Brosens JJ, Cook SJ et al. Paclitaxelinduced nuclear translocation of FOXO3a in breast cancer cells is mediated by c-Jun $\mathrm{NH} 2$ terminal kinase and Akt. Cancer Res 2006; 66: 212-220.

47. Liu B, Han M, Sun RH, Wang JJ, Zhang YP, Zhang DQ et al. ABL-N-induced apoptosis in human breast cancer cells is partially mediated by c-Jun $\mathrm{NH} 2$-terminal kinase activation. Breast Cancer Res 2010; 12: R9.

Cell Death and Disease is an open-access journal published by Nature Publishing Group. This work is licensed under the Creative Commons Attribution-Noncommercial-No Derivative Works 3.0 Unported License. To view a copy of this license, visit http://creativecommons.org/licenses/by-nc-nd/3.0/

\section{Supplementary Information accompanies the paper on Cell Death and Disease website (http://www.nature.com/cddis)}

